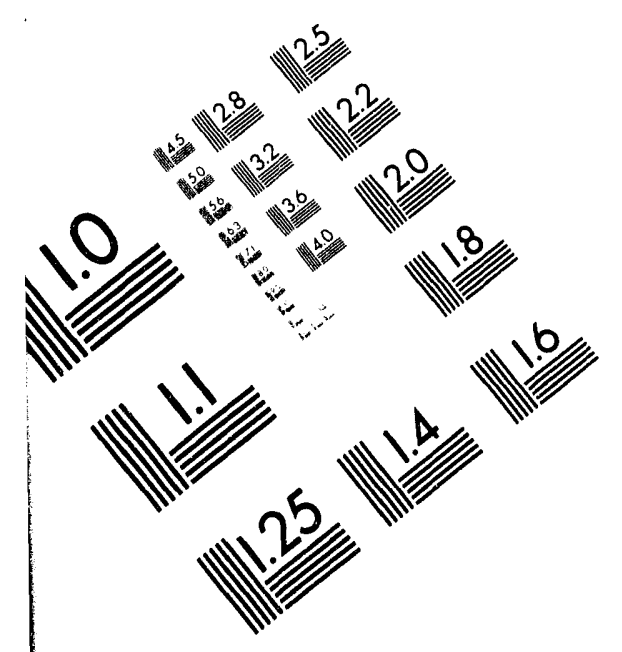

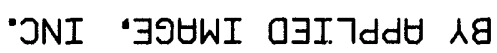

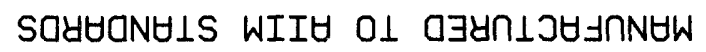
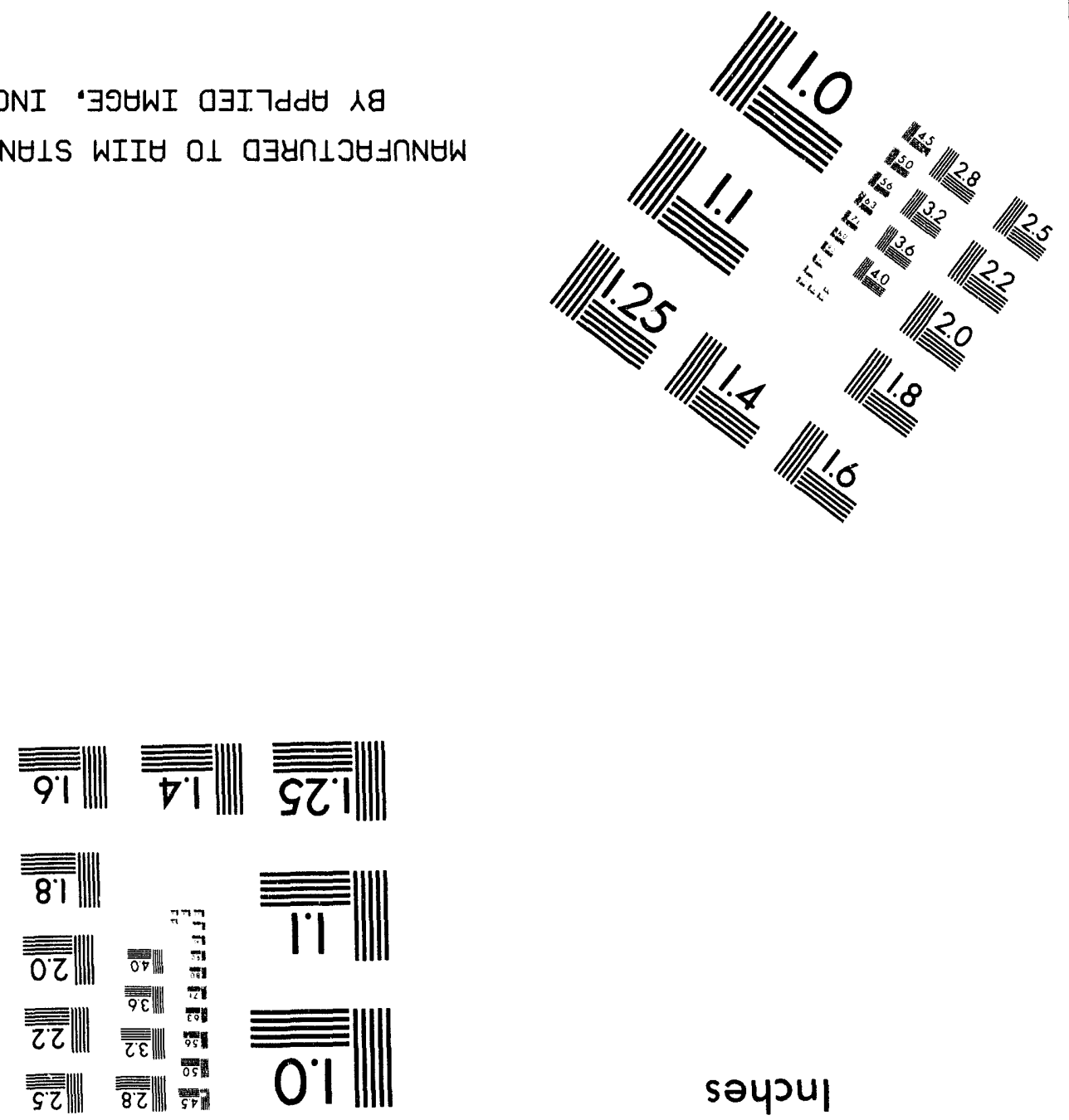

sәчวul

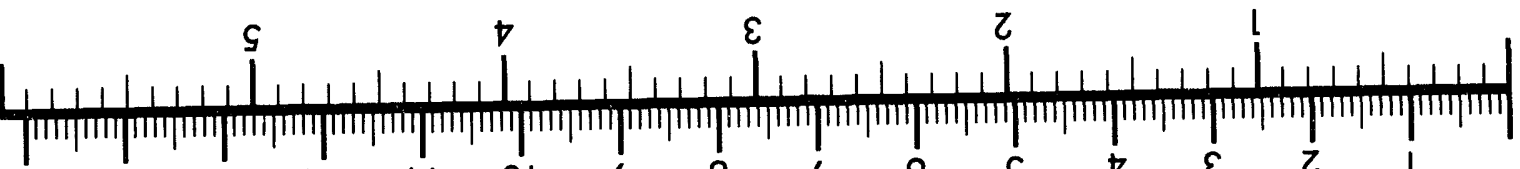

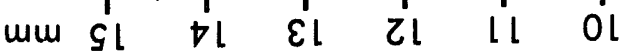

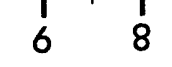

มә†әแ!เนวว

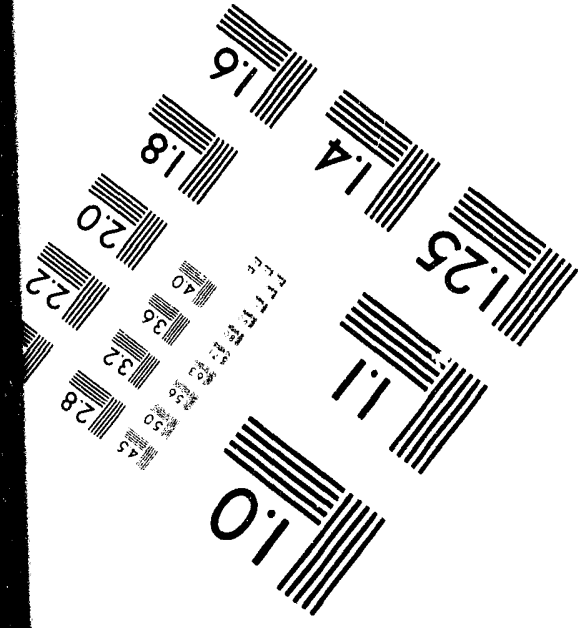

20Z8- L8G/LOE

0เ60z pue|Nuew 'bu!nds jan!!s

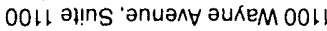

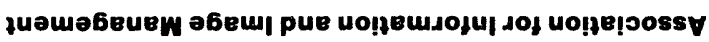

UIIIN

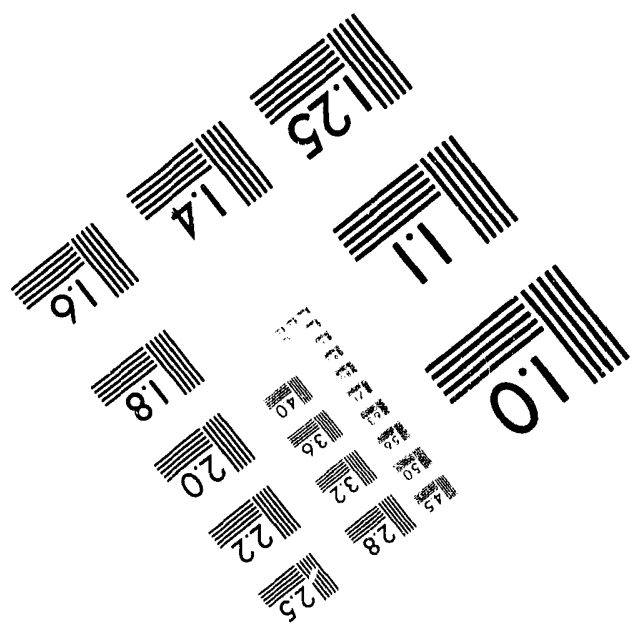



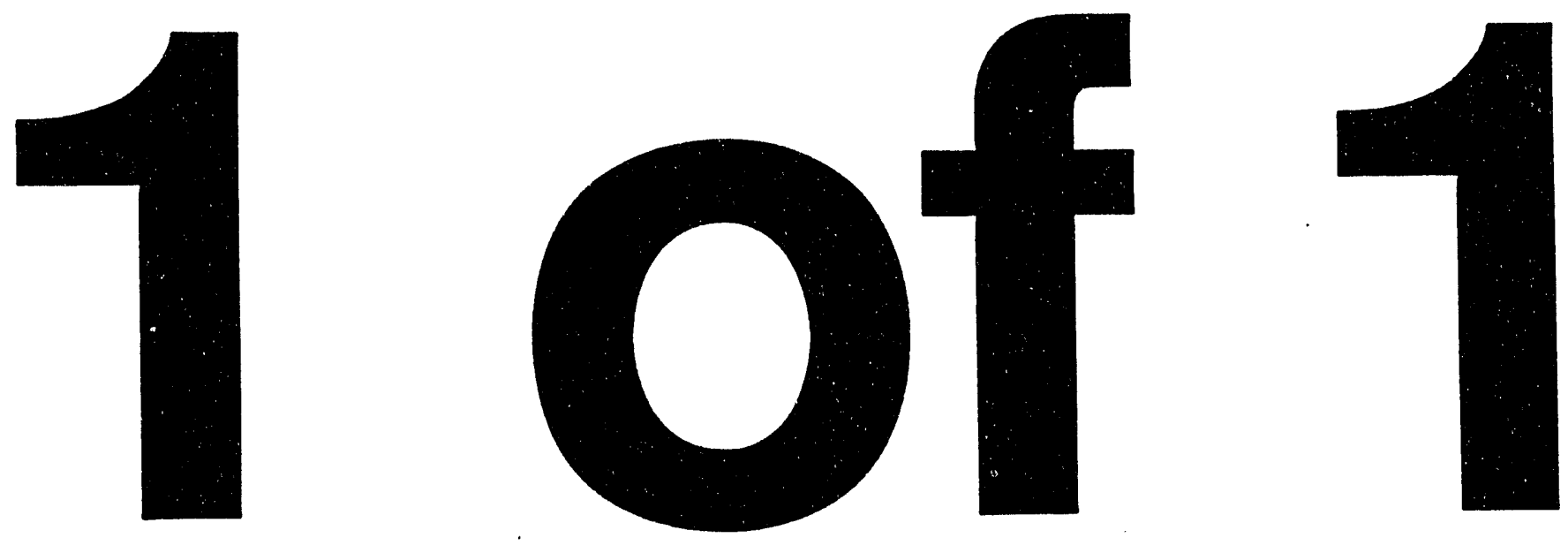


\section{Thermodynamic Characterization of New Palladium Alloy Tritides}

by

J. S. Holder

Westinghouse Savannah River Company

Savannah River Site

Aiken, South Carolina 29808

J. R. Wermer

A document prepared for INTERNATIONAL SYMPOSIUM ON METAL-HYDROGEN SYSTEMS at Fuji-Yoshida, Yamanashi from 11/06/94 - 11/11/94.

\section{DOE Contract No. DE-AC09-89SR18035}

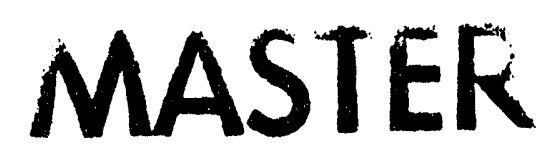

This paper was prepared in connection with work done under the above contract number with the U.S. Department of Energy. By acceptance of this paper, the publisher and/or recipient acknowledges the U.S. Government's right to retain a nonexclusive, royalty-free license in and to any copyright covering this paper, along with the right to reproduce and to authorize others to reproduce all or part of the copyrighted paper. 


\section{DISCLAIMER}

This report was prepared as an account of work sponsored by an agency of the United States Government. Neither the United States Government nor any a'gency thereof, nor any of their employees, makes any warranty, express or implied, or assumes any legal liability or responsibility for the accuracy, completeness, or usefulness of any information, apparatus, product, or process disclosed, or represents that its use would not infringe privately owned rights. Reference herein to any specific commercial product, process, or service by trade name, trademark, manufacturer, or otherwise does not necessarily constitute or imply its endorsement, recommendation, or favoring by the United States Government or any agency thereof. The views and opinions of authors expressed herein do not necessarily state or reflect those of the United States Government or any agency thereof.

This report has been reproduced directly from the best available copy.

Available to DOE and DOE contractors from the Office of Scientific and Technical Information, P. O. Box 62, Oak Ridge, TN 37831; prices available from (615) $576-8401$.

Available to the public from the National Technical Information Service, U. S. Department of Commerce, 5285 Port Royal Rd., Springfield, VA 22161 
Thermodynamic Characterization of New Palladium Alloy Tritides

J. S. Hölder and J. R. Wermer

Westinghouse Savannah River Co., Savannah River Technology Center, Aiken, SC 29808 (USA)

(9 August, 1994)

\begin{abstract}
The decay of tritium in a metal tritide generates ${ }^{3} \mathrm{He}$ in the lattice which tends to degrade the performance of the material over time. It is desired to develop a material which minimizes the tritium aging effects and may be tailored to a particular tritium processing application. Pd alloys with $\mathrm{Ni}$ and $\mathrm{Co}$ have been investigated, as Pd tritide is known to be resistant to tritium aging effects and alloying provides a means for adjusting the plateau pressure of the metal tritide. Sets of tritium desorption isotherms were acquired at temperatures between 273 and $338 \mathrm{~K}$ over the pressure range of 1 to $900 \mathrm{kPa}$. The thermodynamic parameters of $\Delta H$ and $\Delta S$ for the $\beta-\alpha$ phase transition of the metal tritides were determined across the plateau regions of the P-C-T curves. The average values of $\Delta \mathrm{H}(\mathrm{kJ} / \mathrm{mol} \cdot \mathrm{T})$ and $\Delta \mathrm{S}(\mathrm{J} / \mathrm{K} / \mathrm{mol} \cdot \mathrm{T})$ were found to be 15.8 and 50.1 for $\operatorname{Pd}(2.8$ wt.\%)Ni, 13.7 and 50.3 for $\operatorname{Pd}(5.2$ wt.\%)Ni, 15.9 and 51.3 for $\operatorname{Pd}(2.8$ wt.\%)Co, and 13.6 and 51.8 for $\operatorname{Pd}(5.2$ wt.\%)Co, respectively.
\end{abstract}

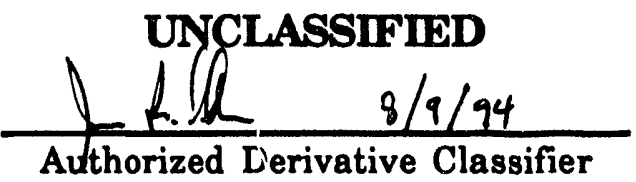




\section{Introduction}

Metal hydride materials are used in a variety of applications at the Savannah River Site (SRS) in the processing of hydrogen isotopes, including: storage, pumping, compression, separation, and purification [1]. For applications involving tritium, the metal tritide must withstand both the processing conditions and the effects of tritium decay. The radiolytic decay of tritium results in the generation of ${ }^{3} \mathrm{He}$ in the metal tritide lattice, which may be retained or released over time. Retention leads to tritium aging effects which may be manifest by a decrease in the equilibrium isotherm plateau pressure, an increase in the plateau slope, a reduction in the reversible storage capacity, and the evolution of a heel [2]. The degree to which, and the rate at which, these effects develop is dependent on the nature of the metal tritide. In tritium processing applications, these factors must be considered for the selection of the metal hydride material.

In the past, tritium research and development of metal hydrides at SRS has focused on $\mathrm{LaNi}_{5}$ based alloys [3]. The substitution of $\mathrm{Al}$ for $\mathrm{Ni}$ in this system tends to stabilize the lattice to tritium aging effects, as well as reducing the equilibrium isotherm pressures of the metal hydride. Thus, $\mathrm{LaNi}_{4.95} \mathrm{Al}_{0.05}$ has been developed for tritium compression, and $\mathrm{LaNi}_{4.25} \mathrm{Al}_{0.75}$ for tritium storage. The extent of aluminum substitution allows the metal hydride pressure to be tailored to the application and reduces tritium aging effects. These benefits are offset by a reduction in the capacity of the material to absorb hydrogen isotopes as the aluminum content is increased.

Current efforts are directed toward developing new palladium alloys for tritium processing applications. Palladium is an excellent storage material, retaining ${ }^{3} \mathrm{He}$ on the order of sixteen years, and developing tritium aging effects slowly over 
time [4,5]. Alloys of palladium, it is thought, should also exhibit good tritium aging behavior, and allow the metal hydride equilibrium pressure to be adjusted by varying the composition of the alloy. Several binary transition metal alloys of palladium, with the formula $\mathrm{Pd}_{\mathbf{x}} \mathrm{M}_{1-\mathrm{x}}(0<\mathrm{x}<0.1)$ and $\mathrm{M}=\mathrm{Rh}, \mathrm{Ni}, \mathrm{Co}$, or $\mathrm{Cr}$, are under study in our laboratory. Selection of the alloying metal, M, and the alloy composition parameter, $x$, determines both the alloy stability to processing conditions, and the thermodynamics of the tritide.

The thermodynamic parameters of change in enthalpy, $\Delta \mathrm{H}$, and change in entropy, $\Delta S$, for the $\beta-\alpha$ phase transition of a metal hydride may be determined from the pressure-composition-temperature (P-C-T) behavior of the system. The plateau pressure in the two phase region of an isotherm (P-C-T curve) is related to the thermodynamics of the phase transition through the following relationship:

$$
\frac{1}{2} \operatorname{Ln}(P)=\frac{\Delta S}{R}-\frac{\Delta H}{R T}
$$

where the plateau pressure $P$ is in atm, the isothermal temperature $T$ is in Kelvin, $R$ is the gas constant, and the factor of $1 / 2$ yields values per mole of atomic hydrogen. From a set of isotherms, acquired at various temperatures, a plot of $\operatorname{Ln}(\mathrm{P})$ versus $1 / \mathrm{T}$ may be constructed and fit to a straight line. A comparison of the line of best fit to equation (1) allows $\Delta H$ and $\Delta S$ to be calculated. The knowledge of $\Delta \mathrm{H}$ and $\Delta \mathrm{S}$ permits the plateau pressure of a hydride to be predicted as a function of temperature, and facilitates the selection of one hydride over another for a particular application. Thus, it is the careful acquisition of P-C-T curves which characterizes the behavior of hydride materials for processing applications. 
Most recently, palladium alloys with $\mathrm{Ni}$ and Co have been characterized by the acquisition of tritium desorption isotherms. Two compositions in each of these alloy systems have been studied, and those results are presented here.

\section{Experimental Details}

\subsection{Materials}

The palladium alloys where supplied by researchers at Los Alamos National Laboratory in the form of annealed foils approximately $100 \mu \mathrm{m}$ thick. The foils were cold rolled from homogenized ingots prepared by arc melting, and annealed at $1100^{\circ} \mathrm{C}$ in air. Alloy compositions of 2.8 and $5.2 \mathrm{wt. \%}$ (nominally 5 and 9 at.\%) of $\mathrm{Ni}$ or $\mathrm{Co}$ in Pd were prepared. The foil samples were sized into approximately $3 \mathrm{x}$ $6 \mathrm{~mm}$ chips, cleaned in a series of solvent baths (hexanes, followed by acetone, and finally methanol), air dried, and loaded into test vessels constructed of $\mathbf{3 1 6}$ stainless steel. Tritium was supplied, free of ${ }^{3} \mathrm{He}$, from a $\mathrm{LaNi}_{4.5} \mathrm{Al}_{0.5}$ storage bed with an isotopic profile of $99 \% \mathrm{~T}, 0.6 \% \mathrm{D}$, and $0.4 \% \mathrm{H}$.

\subsection{Activation}

Sample activation consisted of the following sequence: 1) high temperature evacuation under dynamic vacuum at $425 \mathrm{~K}$ (bake out); 2) $\mathrm{D}_{2}$ absorption and temperature cycling; 3) bake out; 4) $\mathrm{T}_{2}$ absorption and temperature cycling; and 5) bake out. Vacuum was produced by an oil sealed rotary vane pump. Typical pressures obtained at the conclusion of a bake out were $<40 \mu \mathrm{m}$ at temperature. $D_{2}$ and $T_{2}$ absorptions were performed at or above room temperature with the addition of a single aliquot of gas to the system. A few (2 - 4) temperature cycles were performed on each absorption, where the material was desorbed by heating 
to $425 \pm 10 \mathrm{~K}$ and allowed to return to room temperature. Steps (4) and (5) were repeated until consistent absorptions were observed.

\subsection{Tritium Loading}

Samples were loaded with tritium in preparation for desorption via a one-step absorption procedure. Sample vessels were submerged in a constant temperature bath at $\approx 313 \mathrm{~K}$ during these procedures. For each loading, an aliquot of tritium was measured, of sufficient size to tritide the sample into the $\beta$-phase, and added to the test vessel. An internal thermal well with a thermocouple probe was used to monitor the temperature excursion of the sample upon tritiding. The system was allowed to equilibrate for one or more hours while maintaining the sample at constant temperature.

\subsection{Desorption Measurements}

Isothermal tritium desorption measurements were acquired by sequentially removing aliquots of tritium from the gas phase, and re-establishing equilibrium, while maintaining the sample temperature in a constant temperature bath. Aliquots of tritiam were removed from the equilibration volume while the test vessel was valved closed. After a minimum wait time (generally one hour), the system pressure ard temperatures were monitored to determine if equilibrium had been established, before subsequent aliquot removal. The aliquot size was adjust3d throughout the desorption, being larger at pressures above and below the plateau than while traversing the two phase region. Aliquot sizes along the pressure plateau were designed to correspond to composition changes of $\mathrm{T} / \mathrm{M}<$ 0.1 , and wait times were typically doubled for data points in this region. The desorptions were terminated after an equilibrium pressure of less than 20 torr 
was obtained. Thus, the desorption isothermal curves were traced in a stepwise manner, and required 20 - 25 data points to complete.

At the conclusion of each desorption isotherm, the test vessel was removed from the constant temperature bath, wrapped in heating tape, and baked out under vacuum for 2 or more hours at $425 \mathrm{~K}$ to remove any residual tritium from the metal lattice. This treatment was similar to the final bake out at the end of the activation process, and served to prepare the sample for the next tritium loading.

\section{Results}

\subsection{Activation}

The first alloy studied was the $\operatorname{Pd}\left(2.8\right.$ wt.\%)Ni sample. Two $\mathrm{D}_{2}$ absorptions with temperature cycling were performed. The first resulted in a partial loading into the $\beta$-phase after cooling to room temperature from $425 \mathrm{~K}$. In the second, a more complete loading was achieved at room temperature. The kinetics of these absorptions were observed to be slow, and the activation was found to be incomplete from the P-C-T data.

The $\operatorname{Pd}(5.2$ wt.\%)Ni alloy was activated according to the procedure described in section 2.2. The $D_{2}$ absorption was successful at room temperature, although the kinetics were exceedingly slow, requiring several hours to reach equilibrium. Activation with $T_{2}$ was initially performed at room temperature. After three attempts with temperature cycling to $425 \mathrm{~K}$, the material never loaded beyond $75 \%$ of capacity, although the final pressures were greater than the plateau pressure. Three more $\mathrm{T}_{2}$ activations at $313 \mathrm{~K}$ and higher pressures resulted in more complete loadings, with some improvement in the absorption kinetics. 
Activation of the $\operatorname{Pd}\left(2.8\right.$ wt.\%)Co sample was facile. Complete $\mathrm{D}_{2}$ loading at $313 \mathrm{~K}$ occurred on the first attempt. $\mathrm{T}_{2}$ absorption and temperature cycling also resulted in absorption into the $\beta$-phase at $313 \mathrm{~K}$. The kinetics of these processes were improved over that of the Pd alloys with $\mathrm{Ni}$.

The $\operatorname{Pd}(5.2$ wt.\%)Co alloy was activated by our standard procedure, however difficulties were encountered due to the high plateau pressure of this material at $313 \mathrm{~K}$. The $\mathrm{D}_{2}$ absorption at $313 \mathrm{~K}$ resulted in only $\alpha$-phase deuteride formation. $\mathrm{T}_{2}$ activations did not result in $\beta$-phase tritide formation until the sample was temperature cycled between room temperature and $425 \mathrm{~K}$. Seven (7) subsequent absorption/desorption cycles, with vacuum desorptions at $425 \mathrm{~K}$, produced increasing degrees of tritide formation up to a steady state composition of $\mathrm{T} / \mathrm{M}=$ 0.38 at $295 \mathrm{~K}$

\subsection{Tritium Loadings}

The first two (2) loadings of the $\operatorname{Pd}(2.8 \mathrm{wt} . \%) \mathrm{Ni}$ sample were incomplete due to a lack of activation. The initial loading at $295 \mathrm{~K}$ was extremely slow, requiring nearly a day, while the second at $323 \mathrm{~K}$ reach steady state after $5 \mathrm{~h}$. Five (5) more loadings were performed all at $313 \mathrm{~K}$, which yielded complete $\beta$-phase formation with compositions of $T / M=0.59$ in less than $2 \mathrm{~h}$. The internal temperature excursion $\Delta \mathrm{T}$ measured during these absorptions increased from $2 \mathrm{~K}$ in the first loading to $15 \mathrm{~K}$ in the fourth, and remained at that value in later loadings.

The five (5) loadings on the $\operatorname{Pd}(5.2$ wt.\%)Ni sample were all performed at $313 \mathrm{~K}$ in a constant temperature bath. The $\Delta \mathrm{T}$ observed during these procedures was approximately $2 \mathrm{~K}$, and steady state was approached in less than $2 \mathrm{~h}$. The initial loading resulted in a composition of 0.38 , however subsequent absorptions were 
more complete, achieving a $\mathrm{T} / \mathrm{M}=0.45$ or higher. A final loading at $293 \mathrm{~K}$ yielded the largest degree of tritide formation with $\mathrm{T} / \mathrm{M}=0.52$.

The loadings of $\operatorname{Pd}(2.8 \mathrm{wt} . \%) \mathrm{Co}$ sample were consistent and facile, resulting in compositions of $\mathrm{T} / \mathrm{M}=0.51$ at $313 \mathrm{~K}$. Values of $\Delta \mathrm{T}$ ranged from $7 \mathrm{~K}$ on the first absorption to $10 \mathrm{~K}$ on the fourth, while the absorptions were completed in less than $1 \mathrm{~h}$. A fifth loading was completed at $293 \mathrm{~K}$ with a composition of 0.53 .

The initial tritium loading of the $\operatorname{Pd}(5.2$ wt.\%)Co sample was conducted at $296 \mathrm{~K}$, while latter loadings were performed at $273 \mathrm{~K}$. The absorptions required less than $1 \mathrm{~h}$ to complete, however the heat evolution observed in each case was small, with $\Delta \mathrm{T}=2 \mathrm{~K}$. A sample composition of 0.38 was obtained at the higher temperature, while the remaining loadings resulted in $\mathrm{T} / \mathrm{M}$ values between 0.40 and 0.42 .

\subsection{Tritium Desorption Isotherms}

A total of six (6) desorption isotherms were acquired for the $\operatorname{Pd}(2.8$ wt.\%)Ni sample. The isotherms were collected in the following order and temperatures: $303,323,338,338,323$, and $303 \mathrm{~K}$. Equilibration times for the first isotherm were extremely slow, requiring up to $24 \mathrm{~h}$ for data points in the two phase region of the P-C-T curve. The kinetics improved on the second and third isotherms, where equilibrium in the plateau region required approximately 8 and $2 \mathrm{~h}$, respectively, to establish. In the remaining isotherms, the equilibration times on the plateau stabilized at approximately $1.5 \mathrm{~h}$. The two consecutive P-C-T curves collected at $338 \mathrm{~K}$ were coincident, while those measured at $323 \mathrm{~K}$ overlapped from the middle of the plateau to the end of the isotherms in the $\alpha$-phase region. The final data set exhibited a $24.5 \mathrm{kPa}$ increase in the plateau pressure over that observed in the initial isotherm at $303 \mathrm{~K}$. The last three P-C-T curves acquired for this sample are shown in Fig. 1. 
For the $\operatorname{Pd}(5.2$ wt.\%)Ni sample, five (5) isotherms were collected in the following order and temperatures: $323,323,323,308$, and $293 \mathrm{~K}$. The equilibration times for data points along the plateau of the P-C-T curves for this alloy decreased from $2 \mathrm{~h}$, for the first two isotherms, to $1 \mathrm{~h}$, for the remaining isotherms. The plateau pressures for all the desorptions at $323 \mathrm{~K}$ were consistent. The last three P-C-T curves acquire ' for this sample are shown in Fig. 2.

Four (4) isotherms were measured for the $\operatorname{Pd}(2.8 \mathrm{wt} . \%)$ Co alloy in the following order and temperatures: $323,323,338$, and $303 \mathrm{~K}$. The kinetics in the two phase region of the P-C-T curves were consistent in all cases for this sample, where approximately $1 \mathrm{~h}$ was required to establish equilibrium. The two consecutive isotherms collected at $323 \mathrm{~K}$ were coincident. The first, third, and fourth P-C-T curves acquired for this sample are shown in Fig. 3.

For the $\operatorname{Pd}(5.2$ wt.\%)Co sample, tritium desorption isotherms were measured in the following order and temperatures: 293,293 , and $273 \mathrm{~K}$. In the plateau regions of the isotherms, equilibration times of $1.3 \mathrm{~h}$ were required at $293 \mathrm{~K}$, while the kinetics at $273 \mathrm{~K}$ required $2 \mathrm{~h}$ to equilibrate the system. The P-C-T curves collected at $293 \mathrm{~K}$ were well correlated throughout the entire composition range investigated. The second and third P-C-T curves measured for this sample are shown in Fig. 4.

\subsection{Thermodynamics}

The thermodynamic parameters, $\Delta H$ and $\Delta S$, for the $\beta-\alpha$ phase transitions in the Pd alloy tritides were determined by constructing van't Hoff plots from the P-C-T data. In Figs. 5 and 6, van't Hoff plots corresponding to the plateau midpoints are shown with the lines of best fit for the 2.8 and $5.2 \mathrm{wt} . \%$ alloys. For each data point in these plots, the pressure of tritium in equilibrium with the sample at the 
midpoint composition was interpolated from the isothermal measurements. The equations of the lines of best fit were compared with equation (1) to calculate the values of $\Delta \mathrm{H}$ and $\Delta \mathrm{S}$.

The isotherms shown in Figs. 1 - 4 were used to construct van't Hoff plots and calculate $\Delta H$ and $\Delta S$ for each sample at three compositions along the plateaus. The plateau midpoint composition was used, along with two other compositions, equispaced below and above the midpoint, on the $\alpha$-and $\beta$-sides of the plateau. These values are listed in Table 1, where "low", "mid", and "high" correspond to the $\alpha$-side, midpoint, and $\beta$-side compositions, respectively.

\section{Discussion}

Each of the four Pd alloys investigated behaved uniquely with respect to activation, loading, and isothermal tritium desorption. However, experience gained on each sample increased the efficiency of the investigation on the remaining alloys, and served to fine tune the experimental techniques.

The activation procedure employed on the $\operatorname{Pd}(2.8$ wt.\%)Ni alloy was insufficient. The sample did not become fully activated until the fourth isotherm, where the kinetics of absorption and desorption stabilized, and the P-C-T behavior became reproducible. For the $\operatorname{Pd}(5.2$ wt.\%)Ni alloy, a much more extensive activation procedure was used, and the absorption temperature was raised in an attempt to increase the kinetics. This stabilized the plateau pressure, however the kinetics continued to improve until the third isotherm. The $\operatorname{Pd}(2.8$ wt.\%)Co alloy became active on its first exposure to $D_{2}$, and showed no increase in kinetics with absorption/desorption cycling. The $\operatorname{Pd}(5.2$ wt.\%)Co alloy proved more difficult to activate, mainly due to its relatively high plateau pressure. This sample also exhibited a gradual increase in tritium absorption capacity with cycling, which 
may be attributed to a lowering of the absorption plateau pressure. In general, activation was more facile for the Co alloys than the $\mathrm{Ni}$ alloys, and the higher wt.\% alloys required more absorption/desorption cycling to become fully activated.

As with activation, loading of the Co alloys was easier than for the $\mathrm{Ni}$ alloys, and the higher wt.\% samples required more time to complete their loadings. The sample temperature excursion $\Delta \mathrm{T}$, observed on the internal thermoccuple during the loading process, reflected the degree of activation of the alloy. As the samples became more active, the kinetics of absorption increased, shortening the time required for tritide formation, and thereby increasing $\Delta \mathrm{T}$. This was observed in the $\operatorname{Pd}(2.8 \mathrm{wt} . \%) \mathrm{Ni}$ alloy, where $\Delta \mathrm{T}$ steadily increased to a constant value after four (4) loadings. Conversely, the $\operatorname{Pd}(2.8$ wt.\%)Co sample showed little increase in $\Delta \mathrm{T}$ over its loadings, and achieved a constant degree of tritide formation, indicating complete activation initially. The loadings on the $\operatorname{Pd}(5.2 \mathrm{wt} . \%) \mathrm{Ni}$ alloy were all slow and steady with a minimal $\Delta T$, however, a consistent increase in the ultimate composition indicated some further conditioning of the material was occurring. The $\mathrm{Pd}(5.2$ wt.\%)Co sample too showed a minimal $\Delta \mathrm{T}$ on loading, although no further activation of the alloy was observed, as the final compositions achieved were constant.

The P-C-T curves shown in Figs. 1 - 4 demonstrate the well behaved nature of these metal tritides. The pressure plateaus were flat, and the transitions from the two phase region to the single phase regions were smooth. The plateau pressures, at a given temperature, increased with wt.\% for both the $\mathrm{Ni}$ and the $\mathrm{Co}$ alloys. Whereas, the capacities of these materials were reduced by increasing the wt.\% of the alloys. For a constant wt.\% alloy composition, the Co alloys exhibited higher plateau pressures than the $\mathrm{Ni}$ alloys at a given temperature. 
This point is illustrated in Figs. 5 and 6, where van't Hoff plots for the $2.8 \mathrm{wt} . \%$ and $5.2 \mathrm{wt} . \% \mathrm{Pd}$ alloys were calculated at the plateau midpoints. The data from the PdCo alloys lies above that from the PdNi alloys. All the data sets were fit very well to straight lines, given by equation (1), and yielded the thermodynamic parameters for the $\beta-\alpha$ phase transition. Similar calculations were used on the $\alpha$ - and $\beta$-sides of the plateaus to determine $\Delta H$ and $\Delta S$ in these regions. The three values ("low", "mid", and "high") for each parameter were averaged and reported in Table 1 for each Pd alloy, along with the values from a $10 \mu \mathrm{m}$ thick pure Pd foil [6].

Both $\Delta H$ and $\Delta S$ are fairly constant throughout the $\beta-\alpha$ phase transition composition ranges for each alloy. The values of $\Delta \mathrm{H}$ for the $2.8 \mathrm{wt} . \%$ alloys are significantly higher than those of the $5.2 \mathrm{wt} . \%$ alloys, and are approaching the value reported for pure $\mathrm{Pd}$ tritide. This is a consequence of the stability of the tritide which increases, as the wt.\% of the alloy decreases, to a maximum value for pure Pd. Consequently, the plateau pressures at a given temperature increase as the wt.\% of the alloys increase. On the other hand, the values found for $\Delta S$ do not deviate significantly from one alloy to the next, yet they are much higher than that reported for pure Pd tritide. This may be attributable to a greater degree of disorder in the Pd alloy tritides than in pure Pd tritides, for both the $\alpha$ - and $\beta$ phases, although the magnitude of this difference is greater for the $\beta$-phase.

\section{Conclusion}

The annealed Pd alloy foils studied in this investigation were activated, loaded with tritium, and isothermally desorbed at several temperatures to determine their behavior as metal tritides and the thermodynamic parameters of the $\beta-\alpha$ phase transition. The PdCo alloys were activated and loaded with tritium more 
readily than the PdNi alloys, although for both sets of materials, the higher wt.\% alloys were more difficult to activate and slower to load. Absorption/desorption cycling, followed by high temperature desorption under vacuum, proved more effective in activating the samples than temperature cycling in the tritided state. Activation was considered complete when reproducible P-C-T desorption curves were obtained. Although the kinetics of tritide formation and decomposition were not studied quantitatively, qualitative observations showed the kinetics of these processes to improve as the materials became fully activated. The P-C-T behavior of these alloys was typical for metal tritides, with flat pressure plateaus and smooth transitions at the phase boundaries. Plateau pressures increase the wt.\% of the alloys, while the PdCo alloys exhibited higher plateau pressures than the PdNi alloys at a given wt.\% composition. The increased plateau pressures came at the cost of decreased capacity to absorb tritium. The thermodynamic parameters of $\Delta \mathrm{H}$ and $\Delta \mathrm{S}$ for the $\beta-\alpha$ phase transition of the metal tritides were determined from van't Hoff plots of the desorption data in the plateau regions of the P-C-T curves. $\Delta \mathrm{H}$ decreased as the wt.\% of the Pd alloys were increased, however $\Delta \mathrm{S}$ was found to be invariant for the $\mathrm{Pd}$ alloys. The averaged values of $\Delta H$ were determined to be 15.8 and $15.9 \mathrm{KJ} / \mathrm{mol} \mathrm{T}$ for the $2.8 \mathrm{wt} . \%$ alloys, and 13.7 and 13.6 KJ/mol $\mathrm{T}$ for the $5.2 \mathrm{wt. \%}$ alloys of $\mathrm{Pd}$ with $\mathrm{Ni}$ and $\mathrm{Co}$, respectively. The averaged values of $\Delta \mathrm{S}$ were determined to be 50.1 and $51.3 \mathrm{~J} / \mathrm{mol} \mathrm{T}$ for the $2.8 \mathrm{wt} . \%$ alloys, and 51.3 and $51.8 \mathrm{~J} / \mathrm{mol} \mathrm{T}$ for the $5.2 \mathrm{wt} . \%$ alloys of $\mathrm{Pd}$ with $\mathrm{Ni}$ and $\mathrm{Co}$, respectively. Future work involves extending this investigation to $\mathrm{PdCr}$ alloys, as well as studying the tritium aging characteristics of these materials.

\section{Acknowledgments}

The authors would like to thank WSRC Technicians D. E. Moseley and R. J. Stanley for operating the tritium manifold, as well as M. W. Lee of WSRC and 
Prof. Ted Flanagan of the University of Vermont for their fruitful discussions. This work was conducted under the auspices of the Department of Energy under contract DE-AC09-89-SR18035.

\section{References}

1 M. S. Ortman, L. K. Heung, A. Nobile and R.L. Rabun, J. Vac. Sci. Technol. A $8(3)(1990) 2881$.

2 A. Nobile, Jr., R. T. Walters and J. R. Wermer, Fusion Technol. 21 (1992) 769.

3 A. Nobile, Jr., R. T. Walters and W. C. Mosley, J. Less-Common Met., 174 (1991) 1352.

4 J. A. Emig, R. G. Garza, L. D. Christensen, P. R. Coronado and P. C. Souers, J. Nucl. Mater. 187 (1992) 209.

5 R. C. Bowman, Jr., G. Bambakidis, G. C. Abell, A. Attalla and B. D. Craft, Phys. Rev. B 37 (1988) 9447.

6 R. Lässer and K.-H. Klatt, Phys. Rev. B 28 (1983) 748. 
TABLE 1. Thermodynamic parameters, $\Delta \mathrm{H}$ and $\Delta \mathrm{S}$, for the $\beta-\alpha$ phase transition in Pd alloy tritides determined from van't Hoff plots at three positions along the plateau and their average value.

\begin{tabular}{|c|c|c|c|c|c|}
\hline $\begin{array}{c}\Delta \mathrm{H} \\
(\mathrm{kJ} / \mathrm{mnol} \mathrm{T}) \\
\end{array}$ & $\begin{array}{c}\mathrm{PdNi} \\
(2.8 \text { wt.\%) } \\
\end{array}$ & $\begin{array}{c}\text { PdCo } \\
\text { (2.8 wt.\%) } \\
\end{array}$ & $\begin{array}{c}\mathrm{PdNi} \\
(5.2 \text { wt.\%) }\end{array}$ & $\begin{array}{c}\text { PdCo } \\
\text { (5.2 wt.\%) } \\
\end{array}$ & $\mathbf{P d}^{\mathrm{a}}$ \\
\hline$\Delta \mathrm{H}$ (low) & 15.3 & 15.7 & 13.7 & 13.4 & \\
\hline$\Delta \mathrm{H}(\mathrm{mid})$ & 16.2 & 15.8 & 13.6 & 13.6 & 16.6 \\
\hline$\Delta \mathrm{H}$ (high) & 15.8 & 16.3 & 13.7 & 13.7 & \\
\hline$\Delta \mathrm{H}$ (avg) & 15.8 & 15.9 & 13.7 & 13.6 & \\
\hline \multicolumn{6}{|l|}{$\begin{array}{c}\Delta S \\
(\mathrm{~J} / \mathrm{K} / \mathrm{mol} \mathrm{T}) \\
\end{array}$} \\
\hline$\Delta \mathbf{S}$ (low) & 49.0 & 50.8 & 50.5 & 51.4 & \\
\hline$\Delta S($ mid) & 51.5 & 50.9 & 50.0 & 52.1 & 45.9 \\
\hline$\Delta \mathbf{S}$ (high) & 49.9 & 52.3 & 50.3 & 52.0 & \\
\hline$\Delta S$ (avg) & 50.1 & 51.3 & 50.3 & 51.8 & \\
\hline
\end{tabular}

aRef: R. Lässer and K.-H. Klatt, Phys. Rev. B 28 (1983) 748. 


\section{Figure Captions}

Fig. 1. Tritium desorption isotherms for $\operatorname{Pd}(2.8 \mathrm{wt} . \%) \mathrm{Ni}$ alloy.

Fig. 2. Tritium desorption isotherms for $\operatorname{Pd}(5.2$ wt.\%)Ni alloy.

Fig. 3. Tritium desorption isotherms for $\mathrm{Pd}(2.8 \mathrm{wt} . \%)$ Co alloy.

Fig. 4. Tritium desorption isotherms for $\operatorname{Pd}(5.2$ wt.\%)Co alloy.

Fig. 5. van't Hoff plots and lines of best fit for the $2.8 \mathrm{wt} . \%$ Pd alloys calculated at the plateau midpoints.

Fig. 6. van't Hoff plots and lines of best fit for the $5.2 \mathrm{wt} . \%$ Pd alloys calculated at the plateau midpoints. 


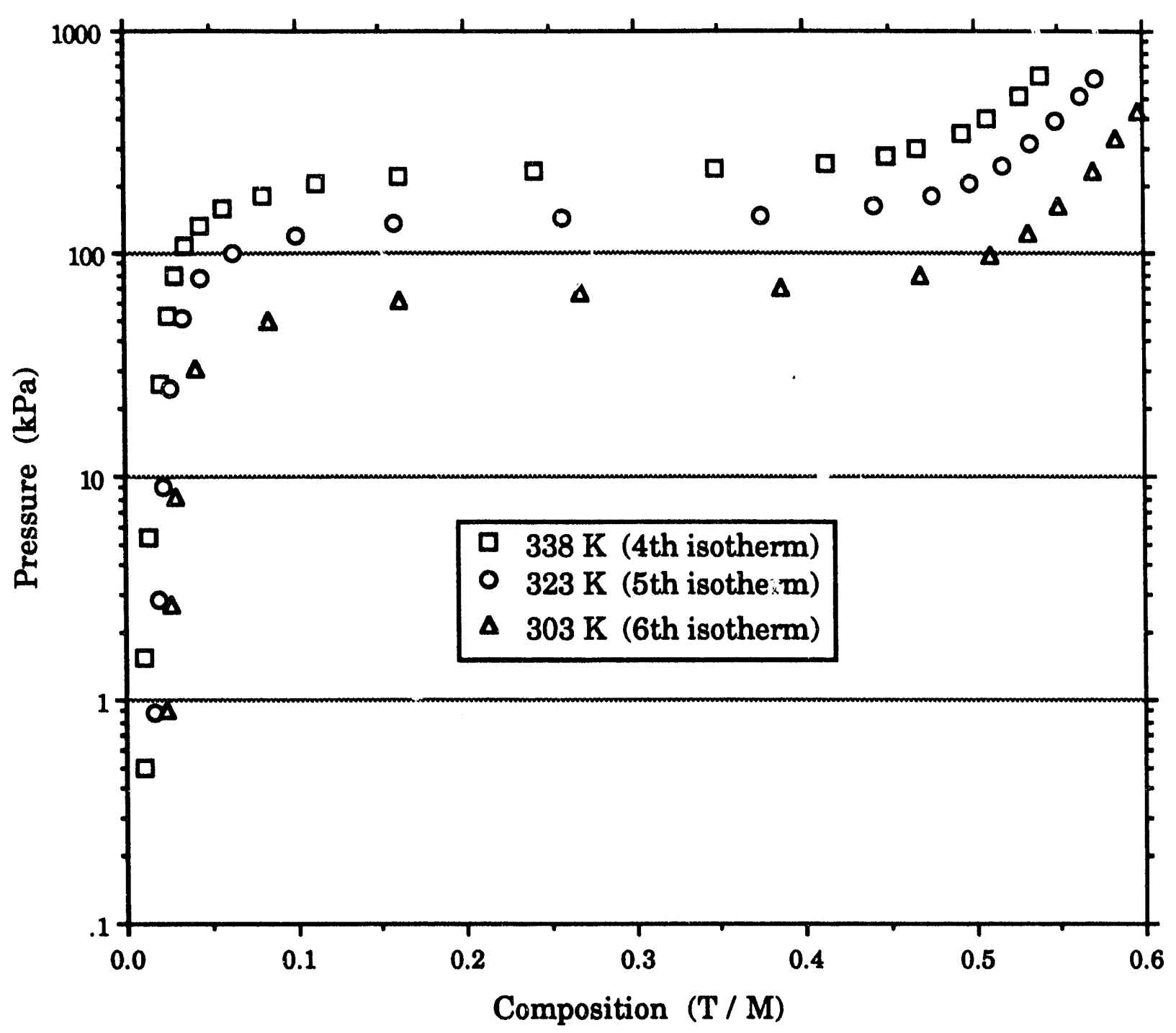

Fig. 1. Tritium desorption isotherms for $\mathrm{Pd}(2.8 \mathrm{wt} . \%) \mathrm{Ni}$ alloy. 


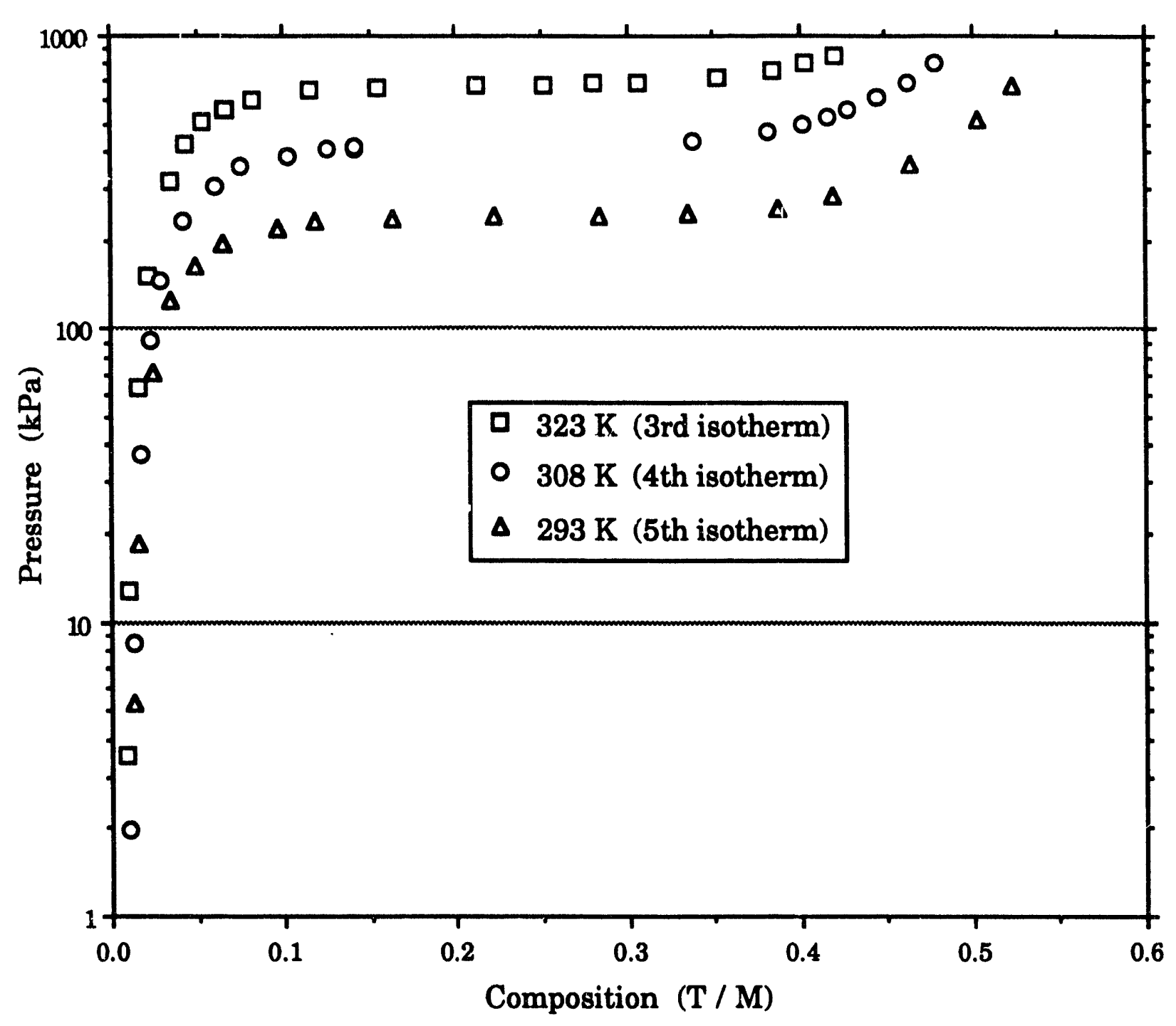

Fig. 2. Tritium desorption isotherms for $\mathrm{Pd}(5.2 \mathrm{wt} . \%) \mathrm{Ni}$ alloy. 


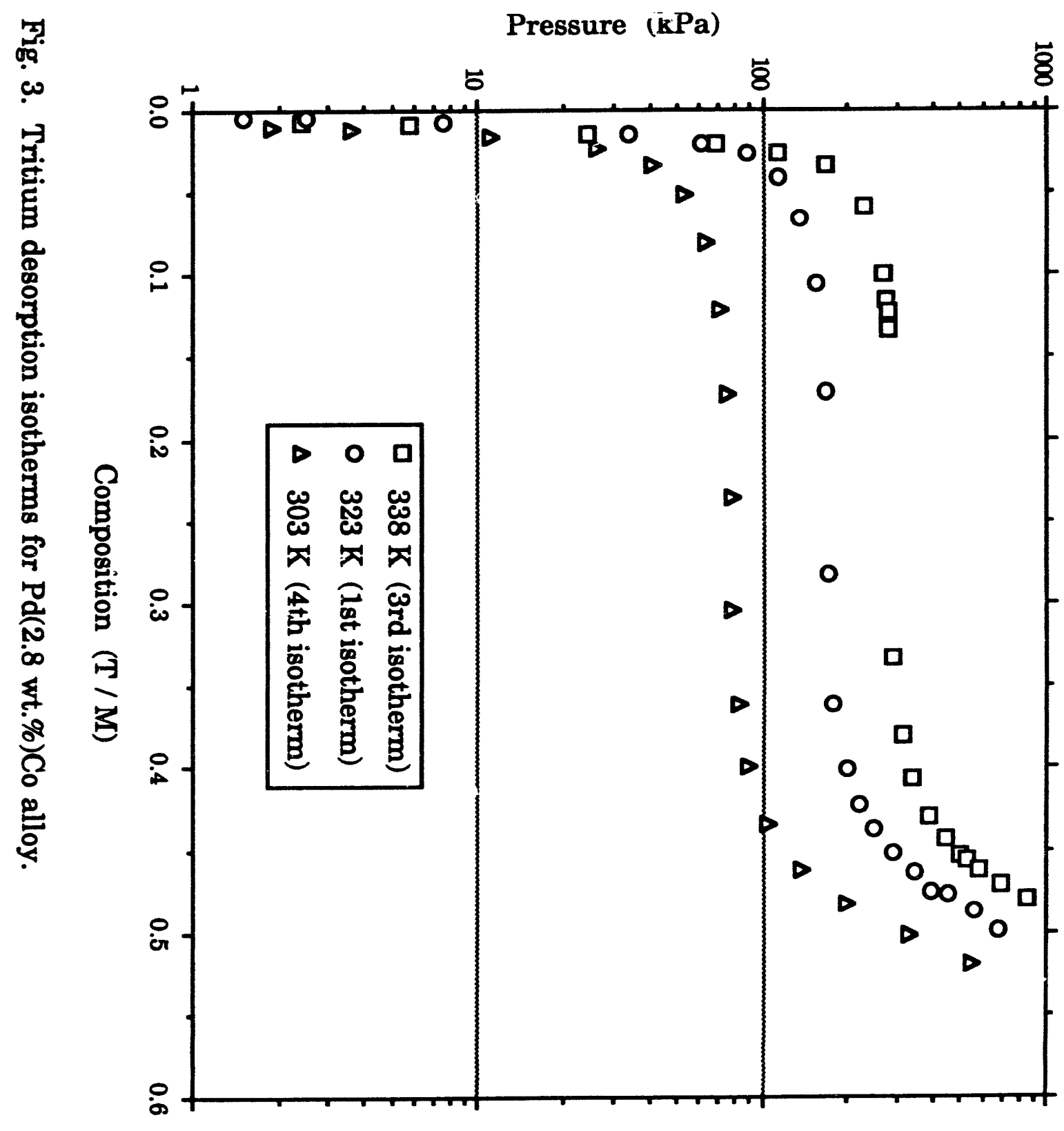




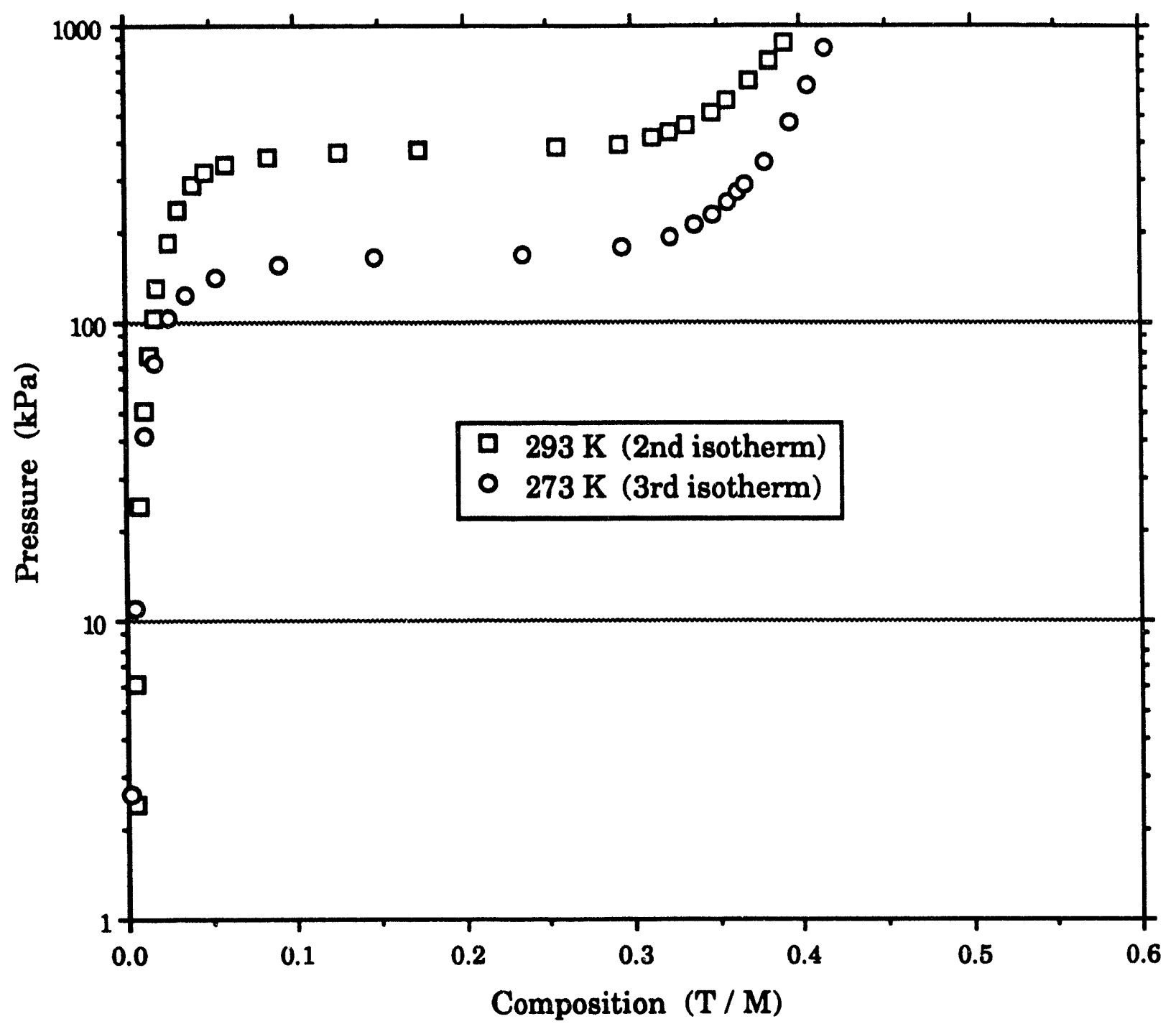

Fig. 4. Tritium desorption isotherms for $\operatorname{Pd}(5.2$ wt.\%)Co alloy. 


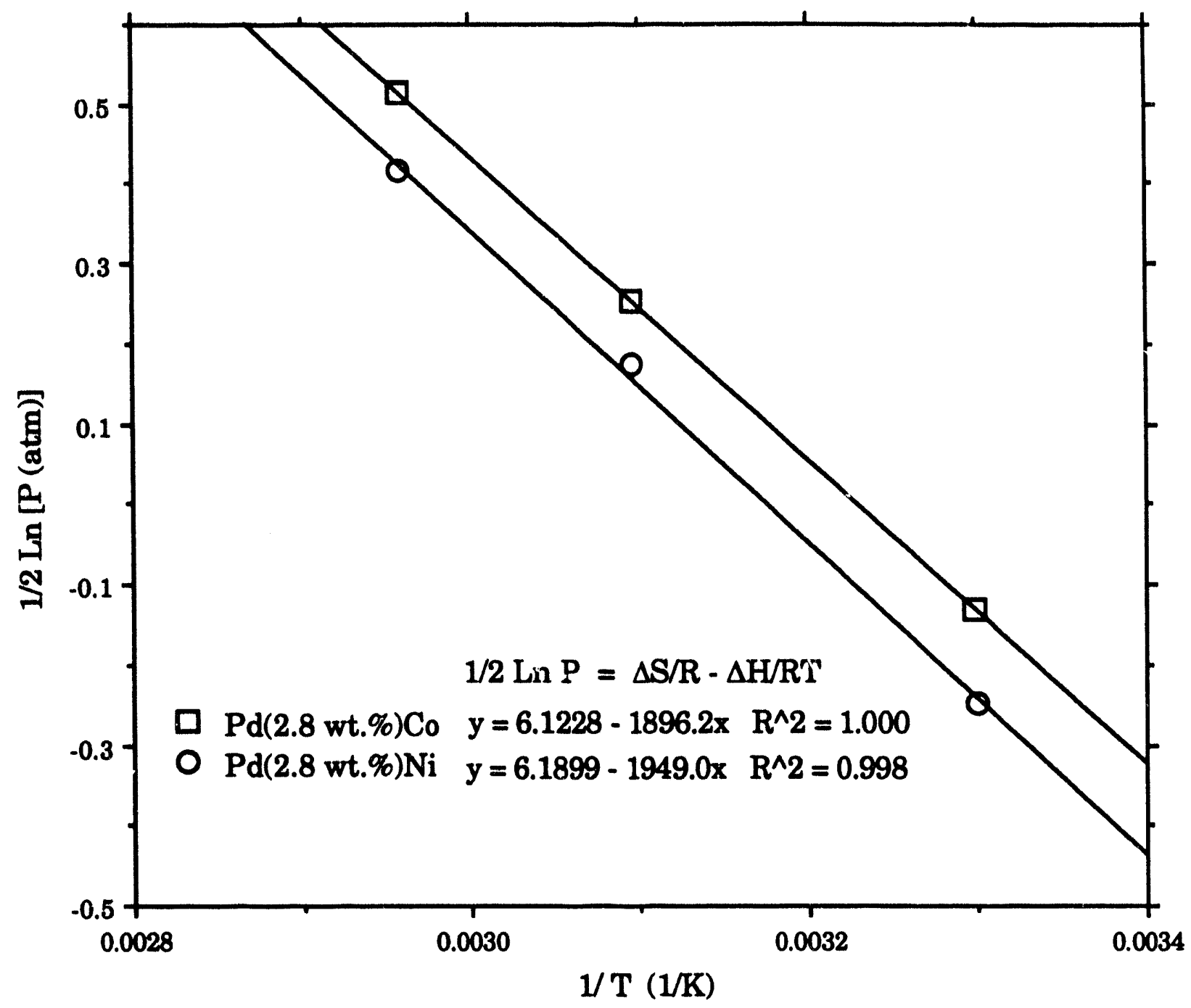

Fig. 5. van't Hoff plots and lines of best fit for the $2.8 \mathrm{wt} . \%$ Pd alloys calculated at the plateau midpoints. 


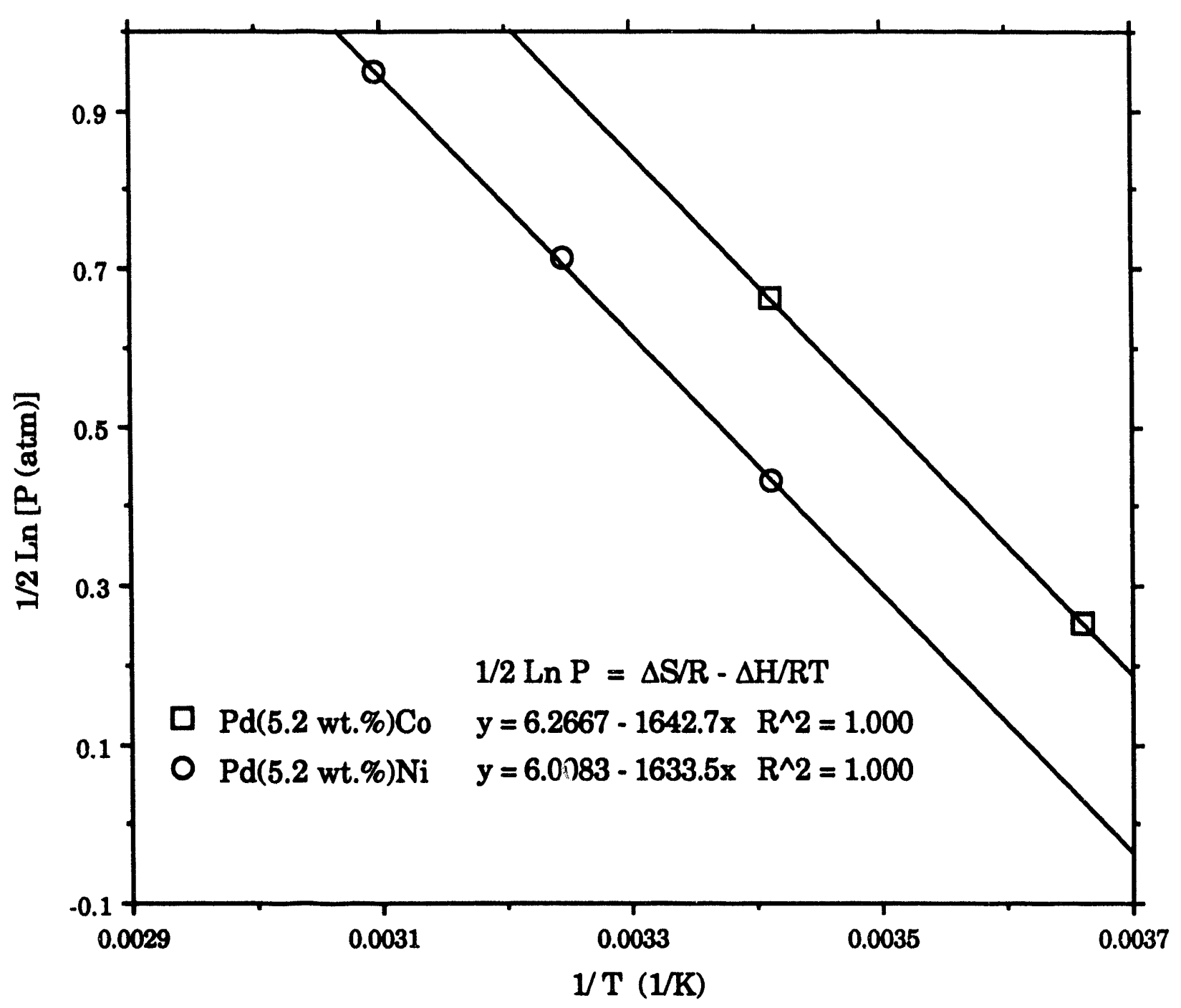

Fig. 6. van't Hoff plots and lines of best fit for the $5.2 \mathrm{wt. \%}$ Pd alloys calculated at the plateau midpoints. 

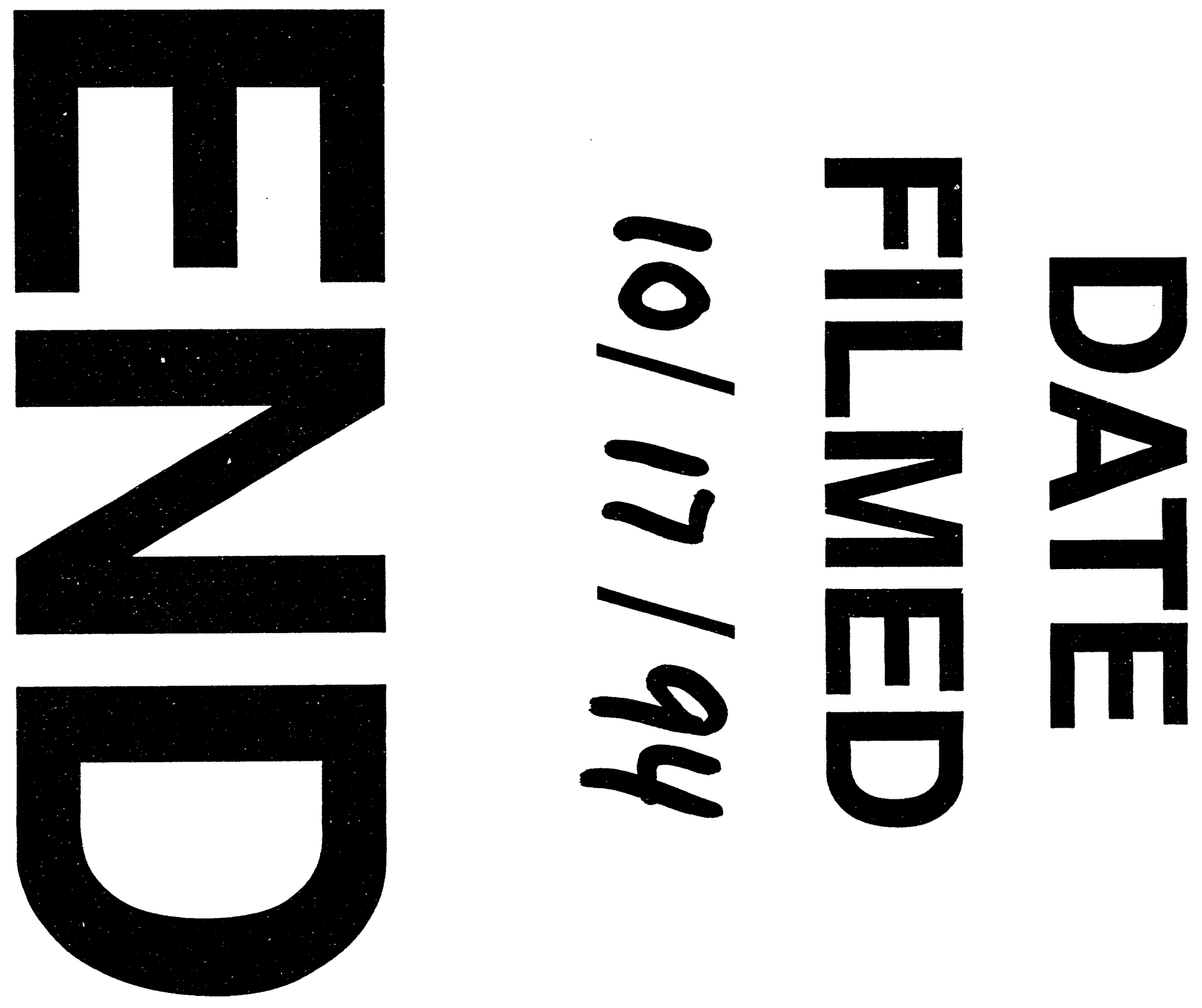